\title{
Polynomial growth harmonic functions on complete Riemannian manifolds
}

Yong Hah Lee

\begin{abstract}
In this paper, we give a sharp estimate on the dimension of the space of polynomial growth harmonic functions with fixed degree on a complete Riemannian manifold, under various assumptions.
\end{abstract}

\section{Introduction}

The classical Liouville theorem, which states that any positive harmonic function on $\mathbb{R}^{2}$ must be constant, has long been an interesting topic of study to analysts and geometers. In 1975, Yau [26] generalized the classical Liouville theorem to complete Riemannian manifolds with nonnegative Ricci curvature. He proved that every positive harmonic function on such a manifold is constant. After the work, he conjectured the following:

Conjecture 1.1 Let $M$ be a complete Riemannian manifold with nonnegative Ricci curvature. Let $r(x)$ denote the distance of any point $x$ from a fixed point o in $M$. Then the space of harmonic functions of polynomial growth of degree at most $d$

$$
\mathcal{H}^{d}(M)=\left\{f: \Delta f=0, \quad|f|(x)=O\left(r^{d}(x)\right) \text { as } r(x) \rightarrow \infty\right\}
$$

must be finite dimensional for any $d \geq 0$.

As partial results, the case of linear growth on this conjecture has been well developed by Cheeger, Colding and Minicozzi II [3], Li and Tam [20, 21], and Wang [25]. Recently, in a series of papers [5]-[11], Colding and Minicozzi II proved that the conjecture is true. To be precise, they prove that the conjecture is also true on a complete Riemannian manifold $M$ if the

2000 Mathematics Subject Classification: Primary 53C21; Secondary 31C05.

Keywords: Polynomial growth harmonic function, volume doubling condition, Poincaré inequality, mean value property. 
manifold satisfies the volume doubling condition and the Poincaré inequality as follows:

$(\mathbf{V})$ there exists a constant $\nu>0$ such that for any $x \in M$ and $0<s \leq r$,

$$
V_{x}(r) \leq\left(\frac{r}{s}\right)^{\nu} V_{x}(s)
$$

where $V_{x}(r)$ denotes the volume of the geodesic ball $B_{r}(x)$;

(P) there exists a constant $C>0$ such that for any $x \in M$ and $r>0$,

$$
\int_{B_{r}(x)} f^{2} \leq C r^{2} \int_{B_{r}(x)}|\nabla f|^{2}
$$

where $f \in C^{\infty}\left(B_{r}(x)\right)$ satisfying $\int_{B_{r}(x)} f=0$.

It is well known that these properties are valid on any manifold with nonnegative Ricci curvature with $\nu$ being the dimension of the manifold. Colding and Minicozzi II [10] and Li [19] also considered manifolds satisfying weaker conditions as follows:

(W) there exist constants $C>0$ and $\nu>0$ such that for any $x \in M$ and $0<s \leq r$,

$$
V_{x}(r) \leq C\left(\frac{r}{s}\right)^{\nu} V_{x}(s)
$$

(M) there exists a constant $\lambda>0$ such that for any $x \in M$ and $r>0$, any nonnegative subharmonic function $f$ on $M$

$$
f(x) \leq \frac{\lambda}{V_{x}(r)} \int_{B_{r}(x)} f
$$

They proved that on a complete Riemannian manifold satisfying the conditions $(\mathbf{W})$ and $(\mathbf{M})$, the space of harmonic functions of polynomial growth of fixed degree is finite dimensional. Note that if a manifold satisfies the conditions $(\mathbf{W})$ and $(\mathbf{P})$, then the mean value property $(\mathbf{M})$ also holds on the manifold. (See [14] or [23].) On the other hand, Wang [25] proved, by introducing a new inner product, the finite dimensionality of the space of linear growth harmonic functions on a complete Riemannian manifold with nonnegative Ricci curvature outside a compact set and finite first Betti number. Recently, based on the argument of Li [19], Tam [24] generalized the result of Wang to the case of polynomial growth harmonic functions with any fixed degree. To be precise, he gave a sharp bound on the dimension of the space of harmonic functions of polynomial growth of fixed degree 
on a complete Riemannian manifold, which has Ricci curvature decaying at least quadratically to zero near infinity and whose ends satisfy the volume comparison property, introduced in [21].

In this paper, we will estimate the dimension of the space of polynomial growth harmonic functions with fixed degree on a complete Riemannian manifold $M$ with finitely many ends, each of which satisfies the volume doubling condition, the mean value property, and the finite covering condition as follows:

Let $E$ be an end, explained in Section 2, of $M$ and $o$ be a fixed point in $M$;

$(\mathbf{W})_{0}$ there exist constants $C>0$ and $\nu>0$ such that for all $B_{r}(x) \subset E$ and any $0<s \leq r$

$$
V_{x}(r) \leq C\left(\frac{r}{s}\right)^{\nu} V_{x}(s)
$$

$(\mathbf{M})_{0}$ there exists a constant $\lambda>0$ such that for all $B_{r}(x) \subset E$ and any nonnegative subharmonic function $f$ on $E$,

$$
f(x) \leq \frac{\lambda}{V_{x}(r)} \int_{B_{r}(x)} f ;
$$

$(\mathbf{C})_{0}$ there exist a positive integer $m_{0}$ and points $x_{1}, x_{2}, \ldots, x_{m_{0}}$ in $\partial C_{E, r}$ for all $r>0$ large enough such that

$$
\partial C_{E, r} \subset \bigcup_{j=1}^{m_{0}} B_{r / 4}\left(x_{j}\right)
$$

and $\cup_{j=1}^{m_{0}} B_{r / 4}\left(x_{j}\right)$ is connected, where $C_{E, r}$ denotes the unbounded component of $E \backslash B_{r}(o)$.

Note that these properties are satisfied on a complete Riemannian manifold with nonnegative Ricci curvature outside a compact set and finite first Betti number. We prove in Section 2 that for given $0<\alpha<1 / 4$, if $\left\{x_{1}, x_{2}, \ldots, x_{m(\alpha)}\right\}$ is a maximal set of points in $\partial C_{E, r}$ such that $d\left(x_{i}, x_{j}\right) \geq$ $\alpha r$ for $i \neq j$, then $m(\alpha) \leq C \alpha^{-\nu}$ for some constant $C>0$. Using this result, we have an upper bound of the dimension of the space of polynomial growth harmonic functions as follows:

Theorem 1.2 Let $M$ be a complete Riemannian manifold with finitely many ends $E_{i}, i=1,2, \ldots, l$, each of which satisfies the conditions $(\mathbf{W})_{0},(\mathbf{M})_{0}$ and $(\mathbf{C})_{0}$. Then there is a constant $C>0$ such that for any $d \geq 0$,

$$
\operatorname{dim} \mathcal{H}^{d}(M) \leq C\left(1+\sum_{i=1}^{l} d^{\nu_{i}}\right),
$$

where $\nu_{i}$ denotes the order $\nu$ in $(\mathbf{W})_{0}$ corresponding to each end $E_{i}$. 
The order of $d$ may not be sharp. In Section 3, we consider some specific cases and give a sharp bound of the dimension with respect to each case. One is the case of a connected sum of complete Riemannian manifolds, each of which satisfies the mean value property $(\mathbf{M})$ and the following volume doubling condition:

$\left(\mathbf{V}^{\prime}\right)$ there exist constants $C>0$ and $\nu>0$ such that for any point $x$ and sufficiently large $0<s \leq r$,

$$
V_{x}(r)-V_{x}(s) \leq C\left\{\left(\frac{r}{s}\right)^{\nu}-1\right\} V_{x}(s) .
$$

The condition $\left(\mathbf{V}^{\prime}\right)$ is stronger than $(\mathbf{W})$, but weaker than $(\mathbf{V})$. Therefore, any complete Riemannian manifold with nonnegative Ricci curvature still satisfies the condition $\left(\mathbf{V}^{\prime}\right)$.

Theorem 1.3 Let $M_{i}, i=1,2, \ldots, l$, be complete Riemannian manifolds satisfying the conditions $\left(\mathbf{V}^{\prime}\right)$ and $(\mathbf{M})$. Let $M$ be a connected sum of $M_{1}, M_{2}, \ldots, M_{l}$. Then there is a constant $C>0$ such that for any $d \geq 0$,

$$
\operatorname{dim} \mathcal{H}^{d}(M) \leq C\left(1+\sum_{i=1}^{l} d^{\nu_{i}-1}\right),
$$

where $\nu_{i}$ denotes the order $\nu$ in $\left(\mathbf{V}^{\prime}\right)$ corresponding to $M_{i}$.

Another is the case when $M$ is a complete $n$-dimensional Riemannian manifold with nonnegative Ricci curvature outside a compact set and finite first Betti number. In this case, we prove that for any end $E$ of $M$, if $\left\{x_{1}, x_{2}, \ldots, x_{m(\alpha)}\right\}$ is a maximal set of points in $\partial C_{E, r}$ such that $d\left(x_{i}, x_{j}\right) \geq$ $\alpha r$ for $i \neq j$, then $m(\alpha)=O\left(\alpha^{n-1}\right)$ as $\alpha \rightarrow 0$. From this result, we obtain a sharp estimate of the dimension in such a way that $\operatorname{dim} \mathcal{H}^{d}(M)=O\left(d^{n-1}\right)$ as $d \rightarrow \infty$.

An ingredient exploited in this paper is the concept of the rough isometry which is more general one than the bi-Lipschitz map. In Section 4, we estimate an upper bound of the dimension of the space of polynomial growth harmonic functions on a complete Riemannian manifold being roughly isometric to each case mentioned just above. The key step in doing so is to examine the rough isometric invariance of the conditions needed in obtaining the upper bound of the dimension. For example, one can easily check that the number of ends and the volume doubling condition are invariant under rough isometries between complete Riemannian manifolds. On the other hand, it is not clear whether or not the mean value property for nonnegative subharmonic functions is roughly isometric invariant. Therefore, in order 
to deploy our theory via rough isometry, we assume the Poincaré inequality instead of the mean value property since the Poincaré inequality is a roughly isometric invariant (see [12]). Furthermore, as mentioned above, the mean value property follows from the volume doubling condition and the Poincaré inequality. By using these facts, we will prove that if a complete Riemannian manifold $M$ is roughly isometric to a connected sum $N$ of complete Riemannian manifolds satisfying the volume doubling condition $\left(\mathbf{V}^{\prime}\right)$ and the Poincaré inequality $(\mathbf{P})$, then $\operatorname{dim} \mathcal{H}^{d}(M)$ is bounded by the same order of $d$ as that of $\operatorname{dim} \mathcal{H}^{d}(N)$. Such an argument can be also applied to the case of a complete Riemannian manifold being roughly isometric to a complete Riemannian manifold with nonnegative Ricci curvature outside a compact set and finite first Betti number.

\section{Volume doubling condition, mean value property and finite covering condition}

We begin with defining ends of a complete Riemannian manifold $M$ : Fix a point $o \in M$. We denote by $\sharp(r)$ the number of unbounded components of $M \backslash B_{r}(o)$. It is easy to prove that $\sharp(r)$ is nondecreasing in $r>0$. Let $\lim _{r \rightarrow \infty} \sharp(r)=l$, where $l$ may be infinity, then we say that the number of ends of $M$ is $l$. If $l$ is finite, then we can choose $r_{0}>0$ such that $\sharp(r)=l$ for all $r \geq r_{0}$, and there exist mutually disjoint unbounded components $E_{1}, E_{2}, \ldots, E_{l}$ of $M \backslash B_{r_{0}}(o)$. We call each $E_{i}$ an end of $M$ for $i=1,2, \ldots, l$.

Through this section, $M$ is assumed to be a complete Riemannian manifold with finitely many ends $E_{1}, E_{2}, \ldots, E_{l}$, each of which satisfies the conditions $(\mathbf{W})_{0},(\mathbf{M})_{0}$ and $(\mathbf{C})_{0}$, and we denote by $\nu_{i}$ the order $\nu$ in $(\mathbf{W})_{0}$ corresponding to each end $E_{i}$.

Suppose that $\mathcal{D}$ is a rank $k$ vector bundle over $M$ with a metric. Fix $0<\alpha<1 / 4$. Then by the conditions $(\mathbf{W})_{0}$ and $(\mathbf{C})_{0}$, for each end $E_{i}$ and any $r>r_{0}$, there exists a maximal set $\left\{x_{1}^{i}, x_{2}^{i}, \ldots, x_{m_{i}(\alpha)}^{i}\right\}$ of points in $\partial C_{E_{i}, r}$ such that $d\left(x_{j}^{i}, x_{k}^{i}\right) \geq \alpha r / 4$ if $j \neq k$, where $m_{i}(\alpha)$ is independent of $r$. We now define a positive semidefinite symmetric bilinear form $S_{r}$ on the space of sections $\Gamma(\mathcal{D})$ of $\mathcal{D}$ by

$$
S_{r}(u, v)=\frac{1}{\operatorname{vol} A_{r}} \int_{A_{r}}\langle u, v\rangle+\sum_{i=1}^{l} \sum_{j=1}^{m_{i}(\alpha)} \frac{1}{V_{x_{j}^{i}}(\alpha r)} \int_{B_{\alpha r}\left(x_{j}^{i}\right)}\langle u, v\rangle,
$$

for $u, v \in \Gamma(\mathcal{D})$, where $A_{r}=\cup_{i=1}^{l}\left(M \backslash C_{E_{i}, r}\right)$.

Applying the argument in [19] to our case, we get the following two lemmas similar to those of [19]. (See also [24].) 
Lemma 2.1 Let $\mathcal{K}$ be an $N$-dimensional subspace of $\Gamma(\mathcal{D})$ such that $\Delta|u|^{2} \geq 0$ for all $u \in \mathcal{K}$. Then there is a constant $C>0$ such that for any $0<\alpha<1 / 4$ and an orthonormal basis $\left\{u_{1}, u_{2}, \ldots, u_{N}\right\}$ for $\mathcal{K}$ with respect to $S_{(1+\alpha) r}$,

$$
\sum_{i=1}^{N} S_{r}\left(u_{i}, u_{i}\right) \leq C\left(1+\sum_{i=1}^{l} m_{i}(\alpha)\right)
$$

for all sufficiently large $r \geq r_{0}$.

Proof. Put $F(x)=\sum_{i=1}^{N}\left|u_{i}\right|^{2}(x)$, then $F$ is subharmonic in $M$. Let $E$ be an end of $M$, and $x_{0}$ be a point in $\partial C_{E,(1+\alpha) r}$ such that

$$
F\left(x_{0}\right)=\sup _{\partial C_{E,(1+\alpha) r}(o)} F .
$$

By [18], one can find an $N \times N$ orthogonal matrix $\left(a_{i j}\right)$ such that $v_{i}=$ $\sum_{j=1}^{N} a_{j i} u_{j}$ and $v_{i}\left(x_{0}\right)=0$ for $i \geq k+1$. Hence $F(x) \leq \sum_{i=1}^{k}\left|v_{i}\right|^{2}\left(x_{0}\right)$ for all $x \in \partial C_{E,(1+\alpha) r}$. By the condition $(\mathbf{M})_{0}$,

$$
\sup _{\partial C_{E,(1+\alpha) r}} F \leq \frac{\lambda}{V_{\alpha(1+\alpha) r / 2}\left(x_{0}\right)} \sum_{i=1}^{k} \int_{B_{\alpha(1+\alpha) r / 2}\left(x_{0}\right)}\left|v_{i}\right|^{2} .
$$

Let $\left\{x_{1}, x_{2}, \ldots, x_{m(\alpha)}\right\}$ be the maximal set of points in $\partial C_{E,(1+\alpha) r}$ chosen in defining $S_{(1+\alpha) r}$. Then there exists a point $x_{j}$ such that

$$
d\left(x_{0}, x_{j}\right) \leq \alpha(1+\alpha) \frac{r}{4} .
$$

Since $B_{\alpha(1+\alpha) r / 2}\left(x_{0}\right) \subset B_{\alpha(1+\alpha) r}\left(x_{j}\right) \subset E$, by the condition $(\mathbf{W})_{0}$ and $(2.1)$,

$$
\sup _{\partial C_{E,(1+\alpha) r}} F \leq \frac{C \lambda}{V_{\alpha(1+\alpha) r}\left(x_{j}\right)} \sum_{i=1}^{k} \int_{B_{\alpha(1+\alpha) r}\left(x_{j}\right)}\left|v_{i}\right|^{2}
$$

for some constant $C>0$. Since $\left\{v_{1}, v_{2}, \ldots, v_{N}\right\}$ is orthonormal with respect to $S_{(1+\alpha) r}, F(x) \leq C k \lambda$ for all $x \in \partial C_{E,(1+\alpha) r}$. Applying the above argument to each end, by the maximum principle, we get the consequence.

Slightly modifying the proof of Lemma 2 in [19], one can easily prove the following lemma:

Lemma 2.2 Let $\mathcal{K}$ be an $N$-dimensional subspace of $\Gamma(\mathcal{D})$ with polynomial growth of degree at most $d$. Then for any $0<\alpha<1 / 4$, there exists $r>r_{0}$ such that if $\left\{u_{1}, u_{2}, \ldots, u_{N}\right\}$ is an orthonormal basis for $\mathcal{K}$ with respect to $S_{(1+\alpha) r}$, then

$$
\sum_{i=1}^{N} S_{r}\left(u_{i}, u_{i}\right) \geq N(1+\alpha)^{-(2 d+1)}
$$


We are now ready to prove Theorem 1.2:

Proof of Theorem 1.2. Let $\mathcal{K}$ be an $N$-dimensional subspace of $\mathcal{H}^{d}(M)$. In the case that $0<d \leq 1$, fix an $0<\alpha<1 / 4$. Combining Lemma 2.1 and Lemma 2.2, we get

$$
N \leq C(1+\alpha)^{2 d+1}\left(1+\sum_{i=1}^{l} m_{i}\right) \leq C(1+\alpha)^{3}\left(1+\sum_{i=1}^{l} m_{i}\right),
$$

where $C>0$ is a constant and $m_{i}=m_{i}(\alpha)$. Hence $N$ is bounded.

Next, consider the case that $d>1$. Put $\alpha=(4 d)^{-1}$. Then by Lemma 2.1, there is a constant $C>0$ such that if $r>r_{0}$ and $\left\{u_{1}, u_{2}, \ldots, u_{N}\right\}$ is an orthonormal basis for $\left(\mathcal{K}, S_{(1+\alpha) r}\right)$, then

$$
\sum_{i=1}^{N} S_{r}\left(u_{i}, u_{i}\right) \leq C\left(1+\sum_{i=1}^{l} m_{i}\right),
$$

where $m_{i}=m_{i}(1 / 4 d)$. On the other hand, by Lemma 2.2, we can find $r>r_{0}$ such that if $\left\{u_{1}, u_{2}, \ldots, u_{N}\right\}$ is an orthonormal basis for $\left(\mathcal{K}, S_{(1+\alpha) r}\right)$, then

$$
\sum_{i=1}^{N} S_{r}\left(u_{i}, u_{i}\right) \geq N(1+1 / 4 d)^{-(2 d+1)} .
$$

Hence we get

$$
N \leq C\left(1+\sum_{i=1}^{l} m_{i}\right)
$$

where $C$ is independent of $d$.

To get the consequence, it suffices to prove that $m_{i} \leq C d^{\nu_{i}}$ for each $i=1,2, \ldots, l$. For the sake of convenience, let us just denote a fixed end by $E$. By the condition $(\mathbf{C})_{0}$, for any $r>r_{0}$, there exists a maximal set $A_{0}=\left\{x_{i}: i=1,2, \ldots, m_{0}\right\}$ of points in $\partial C_{E, r}$ such that $d\left(x_{i}, x_{j}\right) \geq r / 4$ for $i \neq j$ and $\cup_{i=1}^{m_{0}} B_{r / 4}\left(x_{i}\right)$ is connected. For each $i, j \in\left\{1,2, \ldots, m_{0}\right\}$ with $i \leq j$, there exists a chain of balls $B_{r / 4}\left(x_{i}\right), B_{r / 4}\left(x_{i+1}\right), \ldots, B_{r / 4}\left(x_{j}\right)$ of length at most $m_{0}$ such that $B_{r / 4}\left(x_{k}\right) \cap B_{r / 4}\left(x_{k+1}\right) \neq \emptyset$ for $k=i, i+1, \ldots, j-1$. Hence by the condition $(\mathbf{W})_{0}$, we get

$$
V_{x_{i}}(r / 4) \leq C^{m_{0}-1} V_{x_{j}}(r / 4),
$$

for some constant $C>0$.

By adding some points in $\partial C_{E, r}$ to $A_{0}$, we can choose a maximal set $A_{\alpha}=\left\{y_{i}: i=1,2, \ldots, m(\alpha)\right\}$ of points in $\partial C_{E, r}$ such that $d\left(y_{i}, y_{j}\right) \geq \alpha r$ for $i \neq j$. Then for each $y_{i} \in A_{\alpha}$, there exists a point $x_{j_{i}} \in A_{0}$ such that $d\left(y_{i}, x_{j_{i}}\right) \leq r / 4$. 
Let us denote the $\alpha r$-neighborhood of $C_{E, r}$ by $B_{\alpha r}\left(C_{E, r}\right)$. Then by the condition $(\mathbf{W})_{0}$ and $(2.2)$, we get

$$
\begin{aligned}
\operatorname{vol} B_{\alpha r}\left(C_{E, r}\right) & \leq \sum_{x_{j} \in A_{0}} V_{x_{j}}((\alpha+1 / 4) r) \leq m_{0} C^{m_{0}-1} V_{x_{j_{i}}}((\alpha+1 / 4) r) \\
& \leq m_{0} C^{m_{0}-1} V_{y_{i}}((\alpha+1 / 2) r) \leq C \alpha^{-\nu} V_{y_{i}}(\alpha r / 2) .
\end{aligned}
$$

Since $B_{\alpha r / 2}\left(y_{i}\right)$ 's are mutually disjoint, we have

$$
m(\alpha) \operatorname{vol} B_{\alpha r}\left(C_{E, r}\right) \leq C \alpha^{-\nu} \sum_{y_{i} \in A_{\alpha}} V_{y_{i}}(\alpha r / 2) \leq C \alpha^{-\nu} \operatorname{vol} B_{\alpha r}\left(C_{E, r}\right) .
$$

Letting $\alpha=(4 d)^{-1}, m(\alpha) \leq C d^{\nu}$ for some constant $C>0$.

\section{A sharp estimate of the dimension of the space of polynomial growth harmonic function}

In this section, we estimate a sharp bound of the dimension of the space of polynomial growth harmonic functions for some specific cases. We first consider the case of a connected sum of complete Riemannian manifolds satisfying the conditions $\left(\mathbf{V}^{\prime}\right)$ and $(\mathbf{M})$.

Lemma 3.1 Let $M$ be a complete Riemannian manifold satisfying $\left(\mathbf{V}^{\prime}\right)$, and let $o$ be a fixed point in $M$. For any $0<\alpha<1 / 4$ and $r>0$, let $\left\{x_{1}, x_{2}, \ldots, x_{m}\right\}$ be a maximal set of points in $\partial B_{r}(o)$ such that $d\left(x_{i}, x_{j}\right) \geq$ $2 \alpha r$ for $i \neq j$, then $m \leq C \alpha^{-\nu+1}$, where $C$ is independent of $\alpha$ and $r$.

Proof. For any $0<\alpha<1 / 4$ and $r>0$, by $\left(\mathbf{V}^{\prime}\right)$, we have

$$
\begin{aligned}
V_{o}((1+\alpha) r)-V_{o}((1-\alpha) r) & \leq C\left\{\left(\frac{1+\alpha}{1-\alpha}\right)^{\nu}-1\right\} V_{o}((1-\alpha) r) \\
& \leq C \alpha V_{o}(r)
\end{aligned}
$$

Applying $\left(\mathbf{V}^{\prime}\right)$ again, we get

$$
V_{o}(r) \leq V_{x_{i}}(2 r) \leq C\left(\frac{2}{\alpha}\right)^{\nu} V_{x_{i}}(\alpha r)
$$

for each $i=1,2, \ldots, m$. Since $B_{\alpha r}\left(x_{i}\right)$ 's are mutually disjoint, by (3.1)

$$
\begin{aligned}
m V_{o}(r) & \leq C\left(\frac{2}{\alpha}\right)^{\nu} \sum_{i=1}^{m} V_{x_{i}}(\alpha r) \leq C\left(\frac{2}{\alpha}\right)^{\nu}\left(V_{o}((1+\alpha) r)-V_{o}((1-\alpha) r)\right) \\
& \leq C\left(\frac{2}{\alpha}\right)^{\nu} \alpha V_{o}(r) .
\end{aligned}
$$

Hence we have the consequence. 
Using Lemma 3.1, we can prove Theorem 1.3, which is a generalization of the result of [16]:

Proof of Theorem 1.3. We may assume that $M=\cup_{i=1}^{l}\left(M_{i} \backslash K_{i}\right) \cup K$, where $K_{i}$ and $K$ denote some compact subsets of $M_{i}$ and $M$, respectively. For each $i=1,2, \ldots, l$, fix a point $o_{i} \in K_{i}$ and denote by $B_{r}^{i}\left(o_{i}\right)$ the geodesic ball in $M_{i}$ with radius $r$ and center $o_{i}$.

For given $0<\alpha<1 / 4$, choose a maximal set $\left\{x_{1}^{i}, x_{2}^{i}, \ldots, x_{m_{i}}^{i}\right\}$ of points in $\partial B_{r}^{i}\left(o_{i}\right)$ such that $d\left(x_{j}^{i}, x_{k}^{i}\right) \geq \alpha r$ if $j \neq k$. We now define a positive semidefinite bilinear form $S_{r}$ on $\mathcal{H}^{d}(M)$ by

$$
S_{r}(u, v)=\frac{1}{\operatorname{vol} A_{r}} \int_{A_{r}} u v+\sum_{i=1}^{l} \sum_{j=1}^{m_{i}} \frac{1}{V_{x_{j}^{i}}(\alpha r)} \int_{B_{\alpha r}\left(x_{j}^{i}\right)} u v
$$

where $A_{r}=\cup_{i=1}^{l}\left(B_{r}^{i}\left(o_{i}\right) \backslash K_{i}\right) \cup K$ and $r>0$ is sufficiently large. Similarly arguing as in the proof of Theorem 1.2, we get

$$
\operatorname{dim} \mathcal{H}^{d}(M) \leq C\left(1+\sum_{i=1}^{l} m_{i}\right)
$$

Letting $\alpha=(4 d)^{-1}$, by Lemma 3.1, there is a constant $C>0$ such that $m_{i} \leq C d^{\nu_{i}-1}$ for each $i=1,2, \ldots, l$. Hence the result follows.

Next, we consider the case of a complete Riemannian manifold with nonnegative Ricci curvature outside a compact set and finite first Betti number. We introduce a relative volume comparison, which plays a crucial role in obtaining a sharp estimate of the dimension:

Lemma 3.2 Let $M$ be a complete Riemannian manifold with Ricci curvature satisfying $\operatorname{Ric}_{M}(x) \geq-(n-1) K /(1+r(x))^{2}$, where $K$ is a positive constant and $r(x)$ denotes the distance from $x$ to a fixed point $o$ in $M$. Then for any $0<\alpha<1 / 4$ and $r>r_{0}$,

$$
V_{o}((1+\alpha) r)-V_{o}((1-\alpha) r) \leq C \alpha\left(V_{o}((1-\alpha) r)-V_{o}\left(r_{0}\right)\right) .
$$

Proof. Let $g$ be the solution of the linear equation $g^{\prime \prime}=-K g /(1+t)^{2}$ with initial condition $g(0)=0$ and $g^{\prime}(0)=1$. Then

$$
g(t)=\frac{1}{\beta_{1}-\beta_{2}}\left((1+t)^{\beta_{1}}-(1+t)^{\beta_{2}}\right),
$$

where $\beta_{1}=\left(1+(1+4 K)^{1 / 2}\right) / 2$ and $\beta_{2}=\left(1-(1+4 K)^{1 / 2}\right) / 2$. 
By the relative volume comparison in [4], we get

$$
\frac{V_{o}((1+\alpha) r)-V_{o}((1-\alpha) r)}{V_{o}((1-\alpha) r)-V_{o}\left(r_{0}\right)} \leq \frac{\int_{(1-\alpha) r}^{(1+\alpha) r}\left((1+t)^{\beta_{1}}-(1+t)^{\beta_{2}}\right)^{n-1} d t}{\int_{r_{0}}^{(1-\alpha) r}\left((1+t)^{\beta_{1}}-(1+t)^{\beta_{2}}\right)^{n-1} d t}
$$

Since $\beta_{1}>\beta_{2}$ and $r$ is sufficiently large, we get for sufficiently small $\delta>0$

$$
\begin{aligned}
& \frac{V_{o}((1+\alpha) r)-V_{o}((1-\alpha) r)}{V_{o}((1-\alpha) r)-V_{o}\left(r_{0}\right)} \\
& \quad \leq \frac{\left((1+\alpha)^{\beta_{1}(n-1)}-(1-\alpha)^{\beta_{1}(n-1)}\right) r^{\beta_{1}(n-1)}+O\left(r^{\beta_{1}(n-1)-\delta}\right)}{(1-\alpha)^{\beta_{1}(n-1)} r^{\beta_{1}(n-1)}+O\left(r^{\beta_{1}(n-1)-\delta}\right)} \leq C \alpha,
\end{aligned}
$$

where $C$ depends only on $\beta_{1}(n-1)$.

In [22], Liu proved that if $M$ is a complete Riemannian manifold with nonnegative Ricci curvature outside a compact set, then $M$ has only finitely many ends. He also proved that there exist an integer $m_{0}>0$ and points $x_{1}, x_{2}, \ldots, x_{m_{0}}$ in $\partial B_{r}(o)$ such that for each sufficiently large $r>0$,

$$
\partial B_{r}(o) \subset \bigcup_{i=1}^{m_{0}} B_{r / 4}\left(x_{i}\right)
$$

where $o$ is a fixed point in $M$. Hence, for each end $E$ of $M, \partial C_{E, r}$ is also covered by finitely many geodesic balls of radius $r / 4$ with centers in $\partial C_{E, r}$. On the other hand, Li and Tam [21] proved that if $M$ also has finite first Betti number, then each end $E$ of $M$ satisfies the volume comparison property (VC) as follows:

(VC) There is a constant $C>0$ such that for any $r>0$ large enough and any $x \in \partial C_{E, r}$,

$$
\operatorname{vol} A_{r, r_{0}}^{E} \leq C V_{x}(r / 2),
$$

where $A_{r, r_{0}}^{E}$ denotes $\left(B_{r}(o) \backslash B_{r_{0}}(o)\right) \cap E$.

As mentioned above, the conditions $(\mathbf{W})_{0},(\mathbf{M})_{0}$ and $(\mathbf{C})_{0}$ are valid on any complete Riemannian manifold with nonnegative Ricci curvature outside a compact set and finite first Betti number. So, we can apply the argument employed in Section 2 to this case.

Theorem 3.3 Let $M$ be a complete $n$-dimensional Riemannian manifold with nonnegative Ricci curvature outside a compact set and finite first Betti number. Then $M$ has finitely many ends $E_{i}, i=1,2, \ldots, l$, and there is a constant $C>0$ such that for any $d \geq 0$,

$$
\operatorname{dim} \mathcal{H}^{d}(M) \leq C\left(1+\sum_{i=1}^{l} d^{\nu_{i}-1}\right) \leq C\left(1+l d^{n-1}\right),
$$

where $\nu_{i}(\leq n)$ denotes the order $\nu$ in $(\mathbf{W})_{0}$ corresponding to each $E_{i}$. 
Proof. Let $E$ be an end of $M$, then by attaching a compact set $K, E \cup K$ can be regarded as a complete Riemannian manifold with nonnegative Ricci curvature outside a geodesic ball $B_{r_{0}}(o)$ containing $K$ and finite first Betti number. Assume that $E$ satisfies the volume doubling condition $(\mathbf{W})_{0}$ with order $\nu$.

For a sufficiently large $r>r_{0}$, choose a maximal set $A_{0}=\left\{x_{i}: i=\right.$ $\left.1,2, \ldots, m_{0}\right\}$ of points in $\partial C_{E, r}$ such that $d\left(x_{i}, x_{j}\right) \geq r / 4$ for any $i \neq j$. Then $\partial C_{E, r}$ is covered by $B_{r / 4}\left(x_{i}\right)$ 's and $\cup_{x_{i} \in A_{0}} B_{r / 4}\left(x_{i}\right)$ is connected. By the condition ( VC), there exists a constant $C>0$ such that

$$
\operatorname{vol} A_{r, r_{0}}^{E} \leq C V_{x_{i}}(r / 4)
$$

for each $x_{i} \in A_{0}$.

By adding some points to $A_{0}$, we can choose a maximal set $A_{\alpha}=\left\{y_{i}: i=\right.$ $1,2, \ldots, m\}$ of points in $\partial C_{E, r}$ such that $d\left(y_{i}, y_{j}\right) \geq \alpha r$ and $A_{0} \subset A_{\alpha}$. Then for each $y_{j} \in A_{\alpha}$, there exists a point $x_{i_{j}} \in A_{0}$ such that $B_{r / 4}\left(x_{i_{j}}\right) \subset B_{r / 2}\left(y_{j}\right)$. This implies, by the condition $(\mathbf{W})_{0}$, that

$$
V_{x_{i_{j}}}(r / 4) \leq C \alpha^{-\nu} V_{y_{j}}(\alpha r / 2) .
$$

Combining this with (3.2), we get

$$
\operatorname{vol} A_{r, r_{0}}^{E} \leq C \alpha^{-\nu} V_{y_{j}}(\alpha r / 2)
$$

for all $y_{j} \in A_{\alpha}$. Since $\cup_{y_{j} \in A_{\alpha}} B_{\alpha r / 2}\left(y_{j}\right) \subset A_{(1+\alpha) r,(1-\alpha) r}^{E}$, by Lemma 3.2,

$$
\begin{aligned}
m \operatorname{vol} A_{r, r_{0}}^{E} & \leq C \alpha^{-\nu} \operatorname{vol} A_{(1+\alpha) r,(1-\alpha) r}^{E} \\
& \leq C \alpha^{-\nu+1} \operatorname{vol} A_{r, r_{0}}^{E} .
\end{aligned}
$$

Hence $m \leq C \alpha^{-\nu+1}$ for some constant $C>0$.

Since $M$ also satisfies the condition $(\mathbf{M})_{0}$, similarly arguing as in the proof of Theorem 1.2, we get the consequence.

As a simple case, let us consider a connected sum of complete $n$-dimensional Riemannian manifolds with nonnegative Ricci curvature. By the splitting theorem of Cheeger and Gromoll [2], a complete $n$-dimensional Riemannian manifold $M$ with nonnegative Ricci curvature splits isometrically as $\mathbb{R}^{k} \times N^{n-k}$, where $k \leq n$ and $N$ contains no line. Especially, in the case when $M$ is a universal cover of a compact Riemannian manifold with nonnegative Ricci curvature, $N$ becomes a compact set. Since the volume doubling condition $(\mathbf{W})_{0}$ is assumed for sufficiently large radii, the condition $(\mathbf{W})_{0}$ holds on $M$ with the order $\nu=k$. Therefore, it is reasonable to take the order $\nu$ in $(\mathbf{W})_{0}$ being less than or equal to the dimension $n$. Similarly arguing as in the proof of Theorem 3.3, we get the following corollary: 
Corollary 3.4 Let $M$ be a connected sum of complete $n$-dimensional Riemannian manifolds $M_{1}, M_{2}, \ldots, M_{l}$ with nonnegative Ricci curvature. Then for any $d \geq 0$,

$$
\operatorname{dim} \mathcal{H}^{d}(M) \leq C \sum_{i=1}^{l}\left(1+d^{\nu_{i}-1}\right) \leq C l\left(1+d^{n-1}\right),
$$

where $\nu_{i}(\leq n)$ denotes the order $\nu$ in $(\mathbf{W})_{0}$ corresponding to each $M_{i}$.

\section{Rough isometry and polynomial growth harmonic functions}

We begin with introducing the definition of the rough isometry and some local assumptions. A map, not necessarily continuous, $\varphi: X \rightarrow Y$ is called a rough isometry between two metric spaces $X$ and $Y$ if $\varphi$ satisfies the following condition:

(R) for some $\tau>0$, the $\tau$-neighborhood of the image $\varphi(X)$ covers $Y$;

there exist constants $a \geq 1$ and $b \geq 0$ such that

$$
a^{-1} d\left(x_{1}, x_{2}\right)-b \leq d\left(\varphi\left(x_{1}\right), \varphi\left(x_{2}\right)\right) \leq a d\left(x_{1}, x_{2}\right)+b
$$

for all $x_{1}, x_{2} \in X$, where $d$ denotes the distances of $X$ and $Y$ induced from their metrics, respectively.

Especially, being roughly isometric is an equivalence relation. However, since the rough isometry is not necessarily continuous, two roughly isometric metric spaces may have completely different topology. So, in order to deploy our theory via rough isometries between manifolds, it is needed to add the following local assumptions on each manifold:

Let $\varphi: M \rightarrow N$ be a rough isometry satisfying the condition $(\mathbf{R})$.

(i) there exists a constant $C \geq 1$ such that for any point $x \in M$

$$
C^{-1} V_{x}(1) \leq V_{\varphi(x)}(1) \leq C V_{x}(1) ;
$$

(ii) there exists a constant $C_{r}>0$ depending only on $r>0$ such that for any point $x \in M(N$, respectively)

$$
V_{x}(2 r) \leq C_{r} V_{x}(r)
$$

(iii) there exists a constant $C_{r}>0$ depending only on $r>0$ such that for any point $x \in M$

$$
\int_{B_{r}(x)}|f| \leq C_{r} \int_{B_{r}(x)}|\nabla f|,
$$

where $f \in C^{\infty}\left(B_{r}(x)\right)$ with $\int_{B_{r}(x)} f=0$. 
Note that these local assumptions are satisfied on any complete Riemannian manifold with Ricci curvature bounded below by a constant and the positive injectivity radius (see [1], [15] or [12]). From now on, when we say that a map $\varphi: M \rightarrow N$ is a rough isometry between complete Riemannian manifolds $M$ and $N$, it means that the conditions (R), (i), (ii) and (iii) are valid, unless otherwise specified. And $\tau, a$ and $b$ always mean those which appear in $(\mathbf{R})$.

Let us examine whether the properties used in previous sections are invariant under rough isometries between complete Riemannian manifolds. First of all, the number of ends is a rough isometric invariant and, in addition, each rough isometry between manifolds can be reduced to a rough isometry between ends (see [17]). It is easy to prove the rough isometric invariance of the volume doubling condition $(\mathbf{W})_{0}$. Next, let us consider the following Poincaré inequality:

$(\mathbf{P})_{0}$ there exist a constant $C>0$ and an integer $k \in \mathbf{N}$ such that for all $B_{r}(x) \subset E$,

$$
\int_{B_{r / k}(x)} f^{2} \leq C r^{2} \int_{B_{r}(x)}|\nabla f|^{2}
$$

where $f \in C^{\infty}\left(B_{r}(x)\right)$ with $\int_{B_{r / k}(x)} f=0$.

Slightly modifying the argument in [12], one can prove that the condition $(\mathbf{P})_{0}$ is also invariant under rough isometries. Therefore, the mean value property $(\mathbf{M})_{0}$ is valid on any end being roughly isometric to an end satisfying the conditions $(\mathbf{W})_{0}$ and $(\mathbf{P})_{0}$.

We now introduce a modified version of the finite covering condition $(\mathbf{C})_{0}$ as follows:

$\left(\mathbf{C}^{\prime}\right)_{0}$ for any $0<\alpha<1 / 4$ and all $r>r_{0}$ large enough, there exist an integer $m=m(\alpha)$ and points $x_{1}, x_{2}, \ldots, x_{m}$ in $\partial C_{E, r}$ such that

$$
\partial C_{E, r} \subset \bigcup_{i=1}^{m} B_{\alpha r / 2}\left(x_{i}\right)
$$

and $\cup_{i=1}^{m} B_{\alpha r / 2}\left(x_{i}\right)$ is connected.

In [21], Li and Tam proved that if a complete Riemannian manifold $M$ has finitely many ends and finite first Betti number, then each $\partial C_{E, r}$ is connected for sufficiently large $r>0$, where $E$ is an end of $M$. Therefore, if an end of such a manifold $M$ satisfies the condition $(\mathbf{C})_{0}$, then the condition $\left(\mathbf{C}^{\prime}\right)_{0}$ is also valid on the end. Unfortunately, we don't know whether the finite covering condition $\left(\mathbf{C}^{\prime}\right)_{0}$ is a rough isometric invariant. However, we get a modified version of the condition $\left(\mathbf{C}^{\prime}\right)_{0}$ through rough isometries, which is sufficient to take over the role of $\left(\mathbf{C}^{\prime}\right)_{0}$ in proving our result. 
Lemma 4.1 Let $\varphi: D \rightarrow E$ be a rough isometry between ends $D$ and $E$. Suppose that $D$ satisfies the finite covering condition $\left(\mathbf{C}^{\prime}\right)_{0}$. Then for any $0<\alpha<1 / 4$ and all $r>0$ large enough, there exists a sequence $\left\{H_{r}\right\}$ of compact hypersurfaces in $E$ such that $d\left(\partial E, H_{r}\right) \rightarrow \infty$ as $r \rightarrow \infty$,

$$
H_{r} \subset \bigcup_{i=1}^{m} B_{3 a^{2} \alpha r}\left(\varphi\left(x_{i}\right)\right)
$$

and $\cup_{i=1}^{m} B_{3 a^{2} \alpha r}\left(\varphi\left(x_{i}\right)\right)$ is connected, where $m$ and $x_{1}, x_{2}, \ldots, x_{m}$ denote those in $\left(\mathbf{C}^{\prime}\right)_{0}$ corresponding to $D$. In particular, each $H_{r}$ divides $E$ into a bounded subset $K_{r}$ and the unbounded component $U_{r}$ of $E \backslash H_{r}$.

Proof. By the definition of $\varphi$, one can easily prove that $\cup_{i=1}^{m} B_{3 a^{2} \alpha r}\left(\varphi\left(x_{i}\right)\right)$ is connected.

We claim that for sufficiently large $r>0, \cup_{i=1}^{m} B_{3 a^{2} \alpha r}\left(\varphi\left(x_{i}\right)\right)$ divides $E$ into a bounded set and a unbounded component. Otherwise, there exists an arclength parametrized curve $\gamma:[0, \infty) \rightarrow E$ such that $\gamma(0) \in \partial E$, $\gamma(t) \rightarrow \infty$ as $t \rightarrow \infty$ and

$$
B_{c}(\gamma[0, \infty)) \cap \bigcup_{i=1}^{m} B_{2 a^{2} \alpha r}\left(\varphi\left(x_{i}\right)\right)=\emptyset,
$$

where $c=5 a^{2}(\tau+a+b)$. By the definition of $\varphi$, one can choose a sequence $\left\{x_{k}\right\}_{k \in \mathbf{N}}$ in $D$ such that $d\left(\varphi\left(x_{k}\right), \gamma(k)\right) \leq \tau$ for each $k \in \mathbf{N}$. By joining $x_{k}$ and $x_{k+1}$ by minimal geodesic, we get a curve $\sigma:[0, \infty) \rightarrow D$ such that $\sigma(0) \in B_{r_{0}}(\partial D)$ for some $r_{0}>0, \sigma(t) \rightarrow \infty$ as $t \rightarrow \infty$ and

$$
\sigma[0, \infty) \subset \bigcup_{k \in \mathbf{N}} B_{a(2 \tau+b+1)}\left(x_{k}\right) .
$$

By the definition of $\varphi$, we have $\varphi(\sigma[0, \infty)) \subset B_{4 a^{2}(\tau+a+b)}(\gamma[0, \infty))$, hence $B_{\tau}(\varphi(\sigma[0, \infty))) \subset B_{c}(\gamma[0, \infty))$.

On the other hand, $\sigma$ must intersect $\partial C_{D, r}$ at a point $x_{0}$, which implies that $\varphi\left(x_{0}\right) \in B_{c}(\gamma[0, \infty))$. By the definition of $\varphi$, we also have

$$
\varphi\left(\partial C_{D, r}\right) \subset B_{\tau}\left(\varphi\left(\bigcup_{i=1}^{m} B_{\alpha r}\left(x_{i}\right)\right)\right) \subset \bigcup_{i=1}^{m} B_{2 a^{2} \alpha r}\left(\varphi\left(x_{i}\right)\right) .
$$

Hence

$$
\varphi\left(x_{0}\right) \in B_{c}(\gamma[0, \infty)) \cap \bigcup_{i=1}^{m} B_{2 a^{2} \alpha r}\left(\varphi\left(x_{i}\right)\right),
$$

but this contradicts to (4.1). Thus we get the claim. The rest of the proof follows from the condition $(\mathbf{R})$. 
Let $M$ be a complete Riemannian manifold being roughly isometric to a complete Riemannian manifold with ends $D_{i}, i=1,2, \ldots, l$, each of which satisfies the conditions $(\mathbf{W})_{0},(\mathbf{P})_{0}$ and $\left(\mathbf{C}^{\prime}\right)_{0}$. Then $M$ has ends $E_{i}, 1,2, \ldots, l$, and by Lemma 4.1 , each end $E_{i}$ satisfies the following: For any fixed $0<\alpha<1 /\left(40 a^{3}\right)$, there exist an integer $m_{i}=m_{i}(\alpha)$ and points $x_{1}^{i}, x_{2}^{i}, \ldots, x_{m_{i}}^{i}$ such that $d\left(\partial E_{i}, H_{r}^{i}\right) \rightarrow \infty$ as $r \rightarrow \infty$,

$$
H_{r}^{i} \subset \bigcup_{j=1}^{m_{i}} B_{\tilde{\alpha} r}\left(\varphi\left(x_{j}^{i}\right)\right)
$$

and $\cup_{j=1}^{m_{i}} B_{\tilde{\alpha} r}\left(\varphi\left(x_{j}^{i}\right)\right)$ is connected, where $H_{r}^{i}$ divides $E_{i}$ into a bounded subset $K_{r}^{i}$ and the unbounded component $U_{r}^{i}$ of $E^{i} \backslash H_{r}^{i}$, and $\tilde{\alpha}=3 a^{2} \alpha$.

We define a positive semidefinite bilinear form $\tilde{S}_{r}$ on $\mathcal{H}^{d}(M)$ by

$$
\tilde{S}_{r}(u, v)=\frac{1}{\operatorname{vol} A_{r}} \int_{A_{r}} u v+\sum_{i=1}^{l} \sum_{j=1}^{m_{i}} \frac{1}{V_{\varphi\left(x_{j}^{i}\right)}(\tilde{\alpha} r)} \int_{B_{\tilde{\alpha} r}\left(\varphi\left(x_{j}^{i}\right)\right)} u v,
$$

where $A_{r}=\cup_{i=1}^{l} K_{r}^{i}$. It is needed to examine whether the argument used in Section 2 can be well applied to this new bilinear form $\tilde{S}_{r}$.

We claim that for sufficiently large $r>0, \cup_{j=1}^{m_{i}} B_{\tilde{\alpha} r}\left(\varphi\left(x_{j}^{i}\right)\right) \cup K_{r}^{i} \subset K_{(1+\beta) r}^{i}$, where $\beta=10 a^{3} \alpha$. Then we have the same consequence for the case of the new bilinear form $\tilde{S}_{r}$ as that of Lemma 2.1.

Suppose that the claim is not true. Then there exists a point $y \in$ $B_{\tilde{\alpha} r}\left(\varphi\left(x_{j}^{i}\right)\right) \cap B_{\tilde{\alpha}(1+\beta) r}(\varphi(x))$ for some $x_{j}^{i} \in \partial C_{D^{i}, r}$ and $x \in \partial C_{D^{i},(1+\beta) r}$. By the definition of $\varphi$, there exists a point $w \in D^{i}$ such that $d(\varphi(w), y) \leq \tau$, hence $d\left(x_{j}^{i}, w\right) \leq a(\tilde{\alpha} r+b+\tau)$ and $d(x, w) \leq a(\tilde{\alpha}(1+\beta) r+b+\tau)$. This implies that $d\left(x_{j}^{i}, x\right) \leq a \tilde{\alpha}(2+\beta) r+2 a(b+\tau)$, which is impossible, since $x_{j}^{i} \in \partial C_{D^{i}, r}$ and $x \in \partial C_{D^{i},(1+\beta) r}$. Thus we get the claim. One can also easily check the case of Lemma 2.2.

Using the above argument, we have a generalization of the result of Kim and the present author [16] as follows:

Theorem 4.2 Let $M$ be a complete Riemannian manifold roughly isometric to a connected sum of complete Riemannian manifolds $M_{i}, i=1,2, \ldots, l$, each of which satisfies the conditions $\left(\mathbf{V}^{\prime}\right)$ and $(\mathbf{P})$. Then there is a constant $C>0$ such that for any $d \geq 0$,

$$
\operatorname{dim} \mathcal{H}^{d}(M) \leq C\left(1+\sum_{i=1}^{l} d^{\nu_{i}-1}\right)
$$

where $\nu_{i}$ denotes the order $\nu$ in $\left(\mathbf{V}^{\prime}\right)$ corresponding to $M_{i}$. 
Proof. As mentioned above, each end of $M$ satisfies the conditions $(\mathbf{W})_{0}$ and $(\mathbf{P})_{0}$, hence $(\mathbf{M})_{0}$. Applying the argument in Section 2 to the bilinear form $\tilde{S}_{r}$ on $\mathcal{H}^{d}(M)$, for any $0<\alpha<1 /\left(40 a^{3}\right)$,

$$
\operatorname{dim} \mathcal{H}^{d}(M) \leq C\left(1+\sum_{i=1}^{l} m_{i}(\alpha)\right) .
$$

Letting $\alpha=(4 d)^{-1}$, by Lemma 3.1 and Lemma 4.1 , there is a constant $C>0$ such that $m_{i}(1 / 4 d) \leq C d^{\nu_{i}-1}$ for each $i=1,2, \ldots, l$. Hence the result follows.

Corollary 4.3 Let $M$ be a complete Riemannian manifold roughly isometric to a connected sum of complete $n$-dimensional Riemannian manifolds $M_{i}$, $i=1,2, \ldots, l$, with nonnegative Ricci curvature. Then there is a constant $C>0$ such that for any $d \geq 0$,

$$
\operatorname{dim} \mathcal{H}^{d}(M) \leq C\left(1+\sum_{i=1}^{l} d^{\nu_{i}-1}\right) \leq C\left(1+l d^{n-1}\right)
$$

where $\nu_{i}$ denotes the order $\nu$ in $(\mathbf{W})_{0}$ corresponding to $M_{i}$.

Similarly arguing as in Theorem 4.2, together with Theorem 3.3 and Lemma 4.1, we get the following result:

Theorem 4.4 Let $M$ be a complete $n$-dimensional Riemannian manifold with nonnegative Ricci curvature outside a compact set and finite first Betti number. Let $N$ be a complete Riemannian manifold roughly isometric to $M$. Then there is a constant $C>0$ such that for any $d \geq 0$,

$$
\operatorname{dim} \mathcal{H}^{d}(N) \leq C\left(1+\sum_{i=1}^{l} d^{\nu_{i}-1}\right) \leq C\left(1+l d^{n-1}\right)
$$

where $l$ is the number of ends of $M$ and $\nu_{i}$ denotes the order $\nu$ in $(\mathbf{W})_{0}$ corresponding to each end $E_{i}$ of $M$.

Corollary 4.5 Let $M$ be a complete Riemannian manifold with finitely many ends $E_{i}, i=1,2, \ldots, l$. Suppose that each $E_{i}$ is roughly isometric to a Euclidean space $\mathbb{R}^{n_{i}}$. Then there is a constant $C>0$ such that for any $d \geq 0$,

$$
\operatorname{dim} \mathcal{H}^{d}(M) \leq C\left(1+\sum_{i=1}^{l} d^{n_{i}-1}\right)
$$




\section{References}

[1] Buser, P.: A note on the isoperimetric constant. Ann. Sci. École Norm. Sup. (4) 15 (1982), no. 2, 213-230.

[2] Cheeger, J. and Gromoll, D.: The splitting theorem for manifolds of nonnegative Ricci curvature. J. Differential Geom. 6 (1971), 119-128.

[3] Cheeger, J., Colding, T. And Minicozzi II, W.: Linear growth harmonic functions on complete manifolds with nonnegative Ricci curvature. Geom. Funct. Anal. 5 (1995), 948-954.

[4] Cheeger, J., Gromov, M. and Taylor, M.: Finite propagation speed, kernel estimates for functions of the Laplace operator, and the geometry of complete Riemannian manifolds. J. Differential Geom. 17 (1982), 15-53.

[5] Colding, T. and Minicozzi II, W.: On function theory on spaces with a lower Ricci curvature bound. Math. Res. Lett. 3 (1996), 241-246.

[6] Colding, T. And Minicozzi II, W.: Harmonic functions with polynomial growth. J. Differential Geom. 46 (1997), 1-77.

[7] Colding, T. and Minicozzi II, W.: Large scale behavior of kernels of Schrödinger operators. Amer. J. Math. 117 (1997), 1355-1398.

[8] Colding, T. And Minicozzi II, W.: Generalized Liouville properties for manifolds. Math. Res. Lett. 3 (1996), 723-729.

[9] Colding, T. and Minicozzi II, W.: Harmonic functions on manifolds. Ann. of Math. 146 (1997), 725-747.

[10] Colding, T. And Minicozzi II, W.: Liouville theorems for harmonic sections and applications manifolds. Comm. Pure Appl. Math. 51 (1998), $113-138$.

[11] Colding, T. And Minicozzi II, W.: Weyl type bounds for harmonic functions. Invent. Math. 131 (1998), 257-298.

[12] Coulhon, Th. And Saloff-Coste, L.: Variétés riemanniennes isométriques á l'infini. Rev. Mat. Iberoamericana 11 (1995), 687-726.

[13] Grigor'yan, A.: Dimension of space of harmonic functions. Mat. Zametki 48 (1990), 55-61. English translation in Math. Notes 48 (1990), 1114-1118.

[14] Grigor'yan, A.: The heat equation on noncompact Riemannian manifolds. Mat. Sb. 182 (1991) no.1, 55-87. English translation in Math. USSRSb. 72 (1992), no. 1, 47-77.

[15] KanaI, M.: Rough isometries, and combinatorial approximations of geometries of non-compact Riemannian manifolds. J. Math. Soc. Japan $\mathbf{3 7}$ (1985), no. 3, 391-413.

[16] Kim, S. W. And LeE, Y.H.: Polynomial growth harmonic functions on connected sums of complete Riemannian manifolds. Math. Z. 233 (2000), $103-113$. 
[17] Kim, S.W. And LeE, Y.H.: Generalized Liouville property for Schrödinger operator on Riemannian manifolds. Math. Z. 238 (2001), no. 2, $355-387$.

[18] LI, P.: On the Sobolev constant and the $p$-spectrum of a compact Riemannian manifold. Ann. Sci. École Norm. Sup. (4) 13 (1980), no. 4, 451-469.

[19] LI, P.: Harmonic sections of polynomial growth. Math. Res. Lett. 4 (1997), no. $1,35-44$.

[20] Li, P. AND TAM, L. F.: Positive harmonic functions on complete Riemannian manifolds with non-negative curvature outside a compact set. Ann. of Math. (2) 125 (1987), no. 1, 171-207.

[21] LI, P. AND TAM, L. F.: Green's functions, harmonic functions, and volume comparison. J. Differential Geom. 41 (1995), no. 2, 277-318.

[22] LiU, Z-D.: Ball covering property and nonnegative Ricci curvature outside a compact set. In Differential geometry: Riemannian geometry (Los Angeles, CA, 1990), 459-464. Proc. Sympos. Pure Math. 54, Part 3. Amer. Math. Soc., Providence, RI, 1993.

[23] Saloff-Coste, L.: A note on Poincaré, Sobolev and Harnack inequalities. Internat. Math. Res. Notices 1992, no. 2, 27-38.

[24] Tam, L. F.: A note on harmonic forms on complete manifolds. Proc. Amer. Math. Soc. 126 (1998), no. 10, 3097-3108.

[25] WANG, J.: Linear growth harmonic functions on complete manifolds. Comm. Anal. Geom. 3 (1995), no. 3-4, 683-698.

[26] YAU, S. T.: Harmonic functions on complete Riemannian manifolds. Comm. Pure Appl. Math. 28 (1975), 201-228.

Recibido: 31 de julio de 2001

Yong Hah Lee Department of Mathematics Education Ewha Womans University Seoul 120-750, Korea yonghah@ewha.ac.kr 